Supporting Information

\title{
Regioselectivity of $\mathrm{Sc}_{2} \mathrm{C}_{2} @ C_{3 v}(8)-\mathrm{C}_{82}$ : Role of the Sumanene-type Hexagon in Diels-Alder Reaction
}

\author{
Pei Zhao, $\dagger$ Xiang Zhao,,$\dagger$ and Masahiro Ehara $\ddagger$
}

$\dagger$ Institute for Chemical Physics \& Department of Chemistry, State Key Laboratory of Electrical Insulation and Power Equipment, School of Science, Xi'an Jiaotong University, Xi'an 710049, China

\$Institute for Molecular Science, Okazaki 444-8585, Japan

\section{Contents}

I. Figure S1. Representation of the different $[6,6],[5,6]$, and $[5,5]$ bond types that may be present in the fullerene structures, which were first proposed by Poblet and

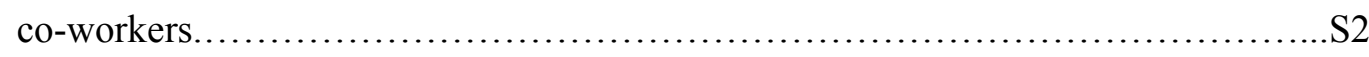

II. Figure S2. The molecular structures of $C_{3 v}(8)-\mathrm{C}_{82}$ and $\mathrm{Sc}_{2} \mathrm{C}_{2} @ C_{3 v}(8)-\mathrm{C}_{82}$ (side

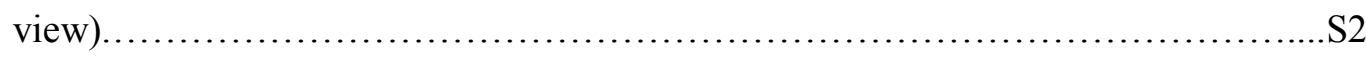

III. Table S1. Relative energies $\left(\Delta \mathrm{E}\right.$, in $\left.\mathrm{kcal} \cdot \mathrm{mol}^{-1}\right)$ of 66 adducts for $\mathrm{Sc}_{2} \mathrm{C}_{2} @ \mathrm{C}_{82}$ in Diels-Alder reaction at the level of M06-2X/3-21G.........................S2

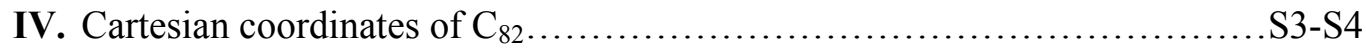

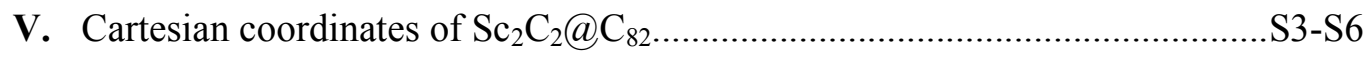

VI. Product and TS from bond 7 of $\mathrm{C}_{82}$ in DA reaction.....................S6-S11

VII. Product and from bond 19 of $\mathrm{Sc}_{2} \mathrm{C}_{2} @ \mathrm{C}_{82}$ in DA reaction............................S11-S15 

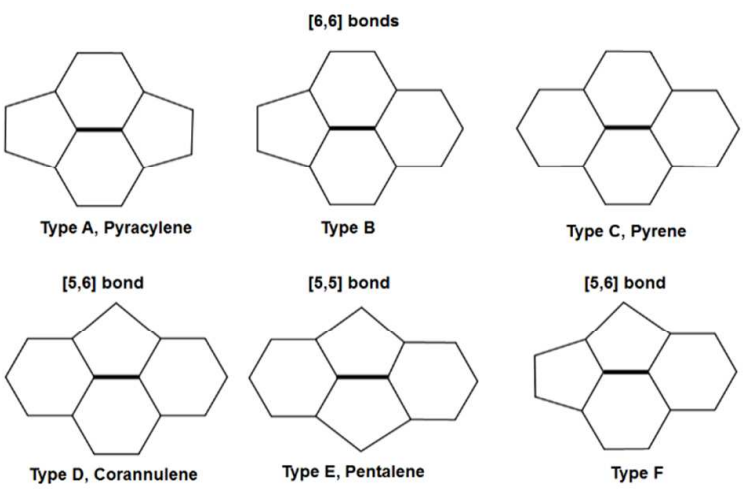

Figure S1. Representation of the different $[6,6],[5,6]$, and $[5,5]$ bond types that may be present in the fullerene structures, which were first proposed by Poblet and co-workers.
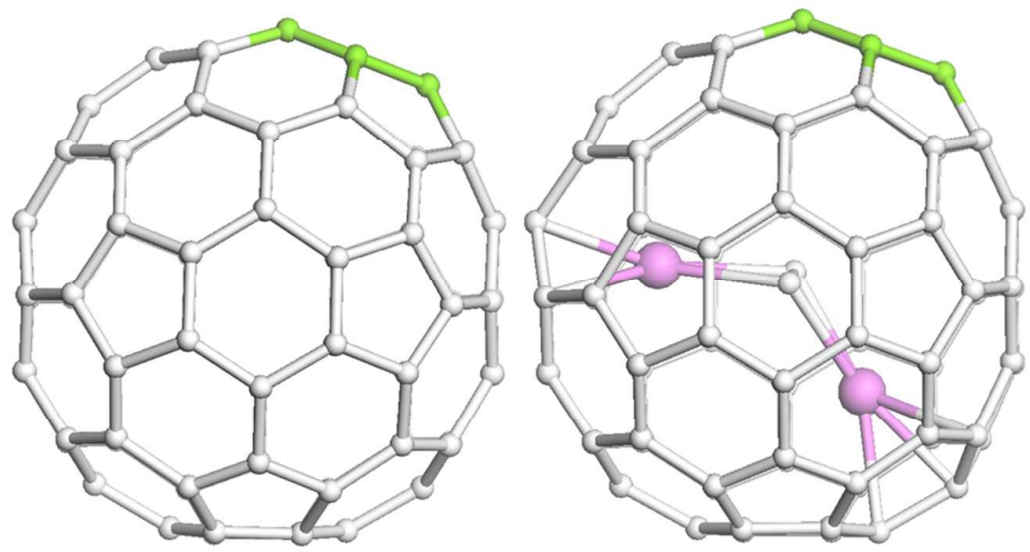

Figure S2. The molecular structures of $C_{3 v}(8)-\mathrm{C}_{82}$ and $\mathrm{Sc}_{2} \mathrm{C}_{2} @ C_{3 v}(8)-\mathrm{C}_{82}$ (side view).

Table S1. Relative energies $\left(\Delta \mathrm{E}\right.$, in $\left.\mathrm{kcal} \cdot \mathrm{mol}^{-1}\right)$ of 66 adducts for $\mathrm{Sc}_{2} \mathrm{C}_{2} @ \mathrm{C}_{82}$ in Diels-Alder reaction at the level of M06-2X/3-21G.

\begin{tabular}{cc|cc|cc|cc|cc|cc}
\hline Bond & $\Delta \mathrm{E}$ & Bond & $\Delta \mathrm{E}$ & Bond & $\Delta \mathrm{E}$ & Bond & $\Delta \mathrm{E}$ & Bond & $\Delta \mathrm{E}$ & Bond & $\Delta \mathrm{E}$ \\
\hline 1 & 20.7 & 5 & 25.5 & 9 & 27.6 & 13 & 28.0 & 17 & 7.8 & 21 & 7.8 \\
1 & 15.9 & 5 & 4.5 & 9 & 17.1 & 13 & 17.0 & 18 & 41.4 & 22 & 14.5 \\
2 & 9.6 & 6 & 9.9 & 10 & 14.7 & 14 & 31.5 & 18 & 35.4 & 22 & 12.2 \\
2 & 11.0 & 6 & 21.1 & 10 & 10.1 & 14 & 39.5 & 18 & 30.9 & 23 & 16.7 \\
2 & 7.6 & 6 & 4.5 & 10 & 16.0 & 15 & 10.3 & 19 & 0.0 & 23 & 19.6 \\
3 & 13.8 & 7 & 2.7 & 11 & 9.6 & 15 & 19.9 & 19 & 1.1 & 23 & 6.8 \\
3 & 14.5 & 7 & 16.2 & 11 & 16.8 & 16 & 7.9 & 20 & 23.4 & 24 & 15.9 \\
3 & 18.7 & 8 & 32.2 & 12 & 23.5 & 16 & 18.5 & 20 & 17.4 & 24 & 17.5 \\
4 & 16.7 & 8 & 22.3 & 12 & 20.2 & 16 & 6.4 & 20 & 17.9 & 24 & 16.4 \\
4 & 21.6 & 8 & 16.8 & 12 & 12.4 & 17 & 14.2 & 21 & 10.6 & 25 & 29.1 \\
5 & 4.5 & 9 & 21.6 & 13 & 29.3 & 17 & 7.6 & 21 & 9.7 & 25 & 31.1 \\
\hline
\end{tabular}


Cartesian coordinates of $\mathrm{C}_{82}, \mathrm{Sc}_{2} \mathrm{C}_{2} @ \mathrm{C}_{82}$ and relevant structures in DA reaction.

$\mathrm{C}_{82}$

C

$\mathrm{C}$

$\mathrm{C}$

$\mathrm{C}$

$\mathrm{C}$

$\mathrm{C}$

$\mathrm{C}$

$\mathrm{C}$

$\mathrm{C}$

$\mathrm{C}$

$\mathrm{C}$

C

C

C

C

C

C

C

C

C

C

C

C

C

C

C

C

C

C

C

C

C

C

C

C

C

C

C

C

C

C

C

$\begin{array}{rrr}-1.895193 & 1.852854 & -3.187569 \\ -1.307359 & 2.968841 & -2.535358 \\ 0.085658 & 3.157554 & -2.517546 \\ 0.433976 & 3.793484 & -1.256020 \\ 1.645675 & 3.541200 & -0.628686 \\ 1.657657 & 3.654838 & 0.814567 \\ 2.568757 & 2.855676 & 1.602478 \\ 1.902044 & 2.386569 & 2.793784 \\ 2.104173 & 1.050601 & 3.255215 \\ 1.013392 & 0.458319 & 3.912141 \\ 0.788757 & -0.973190 & 3.869059 \\ -0.620292 & -1.202149 & 3.924898 \\ -1.212596 & -2.289047 & 3.244106 \\ -2.503734 & -2.079079 & 2.651308 \\ -2.862065 & -2.717481 & 1.383015 \\ -3.720441 & -1.828115 & 0.665594 \\ -3.670069 & -1.752405 & -0.770556 \\ -3.924878 & -0.505745 & -1.373944 \\ -3.269990 & -0.115661 & -2.622611 \\ -3.054073 & 1.260979 & -2.581104 \\ -1.035827 & 1.015158 & -3.916713 \\ -1.261041 & -0.421618 & -3.960358 \\ -2.341740 & -0.997729 & -3.272902 \\ -2.128945 & -2.275653 & -2.691348 \\ -2.800868 & -2.655405 & -1.449571 \\ -1.957153 & -3.523542 & -0.730596 \\ -1.952182 & -3.534093 & 0.704866 \\ -0.704642 & -3.832739 & 1.338692 \\ -0.340043 & -3.207010 & 2.619917 \\ 1.056504 & -3.011665 & 2.632168 \\ 1.652379 & -1.830776 & 3.168599 \\ 2.835229 & -1.257654 & 2.516435 \\ 3.061491 & 0.182994 & 2.559064 \\ 3.816459 & 0.764067 & 1.514378 \\ 3.484522 & 2.075072 & 0.985461 \\ 3.593458 & 2.053053 & -0.476192 \\ 2.633056 & 2.671670 & -1.295982 \\ 2.295622 & 2.032321 & -2.566510 \\ 0.978562 & 2.264088 & -3.163046 \\ 0.374515 & 1.249453 & -3.939334 \\ 1.007608 & -0.036181 & -4.085779 \\ 0.010466 & -1.073596 & -4.009641\end{array}$




\begin{tabular}{|c|c|c|c|}
\hline $\mathrm{C}$ & 0.269092 & -2.267649 & -3.298614 \\
\hline $\mathrm{C}$ & -0.859869 & -2.880766 & -2.697032 \\
\hline $\mathrm{C}$ & -0.733770 & -3.664945 & -1.478347 \\
\hline $\mathrm{C}$ & 0.491676 & -3.831131 & -0.848803 \\
\hline $\mathrm{C}$ & 0.454678 & -4.026061 & 0.585182 \\
\hline $\mathrm{C}$ & 1.559147 & -3.590928 & 1.409471 \\
\hline $\mathrm{C}$ & 2.676123 & -3.093425 & 0.831416 \\
\hline $\mathrm{C}$ & 3.387657 & -1.979771 & 1.433569 \\
\hline $\mathrm{C}$ & 4.100984 & -1.350566 & 0.373962 \\
\hline $\mathrm{C}$ & 4.313546 & 0.009927 & 0.414203 \\
\hline $\mathrm{C}$ & 4.186829 & 0.804080 & -0.806587 \\
\hline $\mathrm{C}$ & 3.865785 & 0.192990 & -2.000530 \\
\hline $\mathrm{C}$ & 2.951050 & 0.823941 & -2.894002 \\
\hline $\mathrm{C}$ & 2.255117 & -0.246114 & -3.586186 \\
\hline $\mathrm{C}$ & 2.584879 & -1.515362 & -2.963049 \\
\hline $\mathrm{C}$ & 1.588512 & -2.483056 & -2.701003 \\
\hline $\mathrm{C}$ & 1.703125 & -3.267442 & -1.473153 \\
\hline $\mathrm{C}$ & 2.799331 & -3.020238 & -0.627332 \\
\hline $\mathrm{C}$ & 3.748806 & -1.995366 & -0.889857 \\
\hline $\mathrm{C}$ & 3.640306 & -1.247009 & -2.043078 \\
\hline $\mathrm{C}$ & -1.842042 & 3.463607 & -1.267312 \\
\hline $\mathrm{C}$ & -0.780901 & 3.989271 & -0.506818 \\
\hline $\mathrm{C}$ & -0.785542 & 3.913691 & 0.927112 \\
\hline $\mathrm{C}$ & 0.487561 & 3.780411 & 1.566287 \\
\hline $\mathrm{C}$ & 0.631960 & 2.998862 & 2.804662 \\
\hline $\mathrm{C}$ & -0.484663 & 2.356788 & 3.383382 \\
\hline $\mathrm{C}$ & -0.259236 & 1.102845 & 3.994669 \\
\hline $\mathrm{C}$ & -1.277855 & 0.080948 & 3.985909 \\
\hline $\mathrm{C}$ & -2.499597 & 0.291770 & 3.334819 \\
\hline $\mathrm{C}$ & -3.123426 & -0.832219 & 2.693914 \\
\hline $\mathrm{C}$ & -3.883204 & -0.663887 & 1.451509 \\
\hline $\mathrm{C}$ & -4.038208 & 0.607252 & 0.845261 \\
\hline $\mathrm{C}$ & -4.122330 & 0.663630 & -0.586012 \\
\hline $\mathrm{C}$ & -3.570665 & 1.759611 & -1.305729 \\
\hline $\mathrm{C}$ & -2.951903 & 2.832480 & -0.634096 \\
\hline $\mathrm{C}$ & -2.989468 & 2.834731 & 0.804478 \\
\hline $\mathrm{C}$ & -1.906724 & 3.375471 & 1.564569 \\
\hline $\mathrm{C}$ & -1.771984 & 2.585177 & 2.790192 \\
\hline $\mathrm{C}$ & -2.743705 & 1.587527 & 2.766036 \\
\hline $\mathrm{C}$ & -3.507868 & 1.732678 & 1.522554 \\
\hline \multicolumn{4}{|c|}{$\mathrm{Sc}_{2} \mathrm{C}_{2} @ \mathrm{C}_{82}$} \\
\hline $\mathrm{C}$ & -1.937079 & 1.902215 & -3.190081 \\
\hline $\mathrm{C}$ & -1.327549 & 2.982525 & -2.485769 \\
\hline $\mathrm{C}$ & 0.109821 & 3.181084 & -2.460139 \\
\hline
\end{tabular}




\begin{tabular}{|c|c|c|c|}
\hline $\mathrm{C}$ & 0.451365 & 3.819906 & -1.239055 \\
\hline $\mathrm{C}$ & 1.696009 & 3.558941 & -0.600557 \\
\hline $\mathrm{C}$ & 1.705338 & 3.664942 & 0.809079 \\
\hline $\mathrm{C}$ & 2.595707 & 2.862325 & 1.590179 \\
\hline $\mathrm{C}$ & 1.910141 & 2.377751 & 2.747311 \\
\hline $\mathrm{C}$ & 2.091849 & 1.053115 & 3.179216 \\
\hline $\mathrm{C}$ & 0.953369 & 0.447701 & 3.807145 \\
\hline $\mathrm{C}$ & 0.734182 & -0.958266 & 3.784060 \\
\hline $\mathrm{C}$ & -0.673646 & -1.206031 & 3.952079 \\
\hline $\mathrm{C}$ & -1.245574 & -2.311108 & 3.211357 \\
\hline $\mathrm{C}$ & -2.519336 & -2.094019 & 2.605251 \\
\hline $\mathrm{C}$ & -2.865321 & -2.715764 & 1.369819 \\
\hline $\mathrm{C}$ & -3.733534 & -1.831679 & 0.654576 \\
\hline $\mathrm{C}$ & -3.689993 & -1.767175 & -0.765028 \\
\hline $\mathrm{C}$ & -3.942802 & -0.504803 & -1.374929 \\
\hline $\mathrm{C}$ & -3.291940 & -0.128641 & -2.593488 \\
\hline $\mathrm{C}$ & -3.067071 & 1.301581 & -2.547345 \\
\hline $\mathrm{C}$ & -1.089506 & 1.049007 & -3.992384 \\
\hline $\mathrm{C}$ & -1.319670 & -0.411493 & -4.040784 \\
\hline $\mathrm{C}$ & -2.396929 & -1.008421 & -3.284224 \\
\hline $\mathrm{C}$ & -2.151504 & -2.262405 & -2.649047 \\
\hline $\mathrm{C}$ & -2.814884 & -2.657650 & -1.446435 \\
\hline $\mathrm{C}$ & -1.941711 & -3.539420 & -0.731394 \\
\hline $\mathrm{C}$ & -1.940610 & -3.563682 & 0.689742 \\
\hline $\mathrm{C}$ & -0.713061 & -3.844278 & 1.328998 \\
\hline $\mathrm{C}$ & -0.366843 & -3.214906 & 2.572066 \\
\hline $\mathrm{C}$ & 1.067591 & -3.013959 & 2.582350 \\
\hline $\mathrm{C}$ & 1.640903 & -1.839131 & 3.098072 \\
\hline $\mathrm{C}$ & 2.830538 & -1.265585 & 2.475884 \\
\hline $\mathrm{C}$ & 3.058112 & 0.183771 & 2.517919 \\
\hline $\mathrm{C}$ & 3.843218 & 0.767705 & 1.500465 \\
\hline $\mathrm{C}$ & 3.538938 & 2.076549 & 0.979167 \\
\hline $\mathrm{C}$ & 3.640286 & 2.058547 & -0.457473 \\
\hline $\mathrm{C}$ & 2.660680 & 2.682946 & -1.265594 \\
\hline $\mathrm{C}$ & 2.301866 & 2.031681 & -2.525974 \\
\hline $\mathrm{C}$ & 0.975941 & 2.267788 & -3.091765 \\
\hline $\mathrm{C}$ & 0.343833 & 1.252304 & -3.895336 \\
\hline $\mathrm{C}$ & 0.973126 & -0.025112 & -4.010036 \\
\hline $\mathrm{C}$ & -0.019004 & -1.052420 & -3.971864 \\
\hline $\mathrm{C}$ & 0.263874 & -2.258743 & -3.235759 \\
\hline $\mathrm{C}$ & -0.847907 & -2.898308 & -2.652663 \\
\hline $\mathrm{C}$ & -0.729952 & -3.683152 & -1.474809 \\
\hline $\mathrm{C}$ & 0.528054 & -3.855800 & -0.833084 \\
\hline $\mathrm{C}$ & 0.490886 & -4.044317 & 0.568293 \\
\hline
\end{tabular}




\begin{tabular}{|c|c|c|c|}
\hline $\mathrm{C}$ & 1.578236 & -3.609863 & 1.389048 \\
\hline $\mathrm{C}$ & 2.722534 & -3.114204 & 0.815669 \\
\hline $\mathrm{C}$ & 3.408391 & -1.997775 & 1.415290 \\
\hline $\mathrm{C}$ & 4.139790 & -1.355577 & 0.359859 \\
\hline $\mathrm{C}$ & 4.352366 & -0.001719 & 0.401666 \\
\hline $\mathrm{C}$ & 4.221566 & 0.796058 & -0.807603 \\
\hline $\mathrm{C}$ & 3.894475 & 0.188862 & -1.995656 \\
\hline $\mathrm{C}$ & 2.951090 & 0.819956 & -2.871393 \\
\hline $\mathrm{C}$ & 2.255488 & -0.241759 & -3.545287 \\
\hline $\mathrm{C}$ & 2.584846 & -1.503531 & -2.943919 \\
\hline $\mathrm{C}$ & 1.592330 & -2.478254 & -2.669089 \\
\hline $\mathrm{C}$ & 1.722103 & -3.283892 & -1.454661 \\
\hline $\mathrm{C}$ & 2.836925 & -3.040217 & -0.617583 \\
\hline $\mathrm{C}$ & 3.780132 & -1.997033 & -0.894468 \\
\hline $\mathrm{C}$ & 3.667165 & -1.247370 & -2.039526 \\
\hline $\mathrm{C}$ & -1.848040 & 3.490384 & -1.255742 \\
\hline $\mathrm{C}$ & -0.752074 & 4.010891 & -0.494303 \\
\hline $\mathrm{C}$ & -0.758308 & 3.950051 & 0.926026 \\
\hline $\mathrm{C}$ & 0.490772 & 3.800846 & 1.567902 \\
\hline $\mathrm{C}$ & 0.614937 & 3.018948 & 2.765574 \\
\hline $\mathrm{C}$ & -0.506815 & 2.383413 & 3.332567 \\
\hline $\mathrm{C}$ & -0.311610 & 1.099782 & 3.965543 \\
\hline $\mathrm{C}$ & -1.353449 & 0.086352 & 4.083792 \\
\hline $\mathrm{C}$ & -2.615687 & 0.314785 & 3.378566 \\
\hline $\mathrm{C}$ & -3.173560 & -0.800258 & 2.666853 \\
\hline $\mathrm{C}$ & -3.910535 & -0.649155 & 1.439070 \\
\hline $\mathrm{C}$ & -4.078190 & 0.624169 & 0.828976 \\
\hline $\mathrm{C}$ & -4.136826 & 0.676172 & -0.591787 \\
\hline $\mathrm{C}$ & -3.581677 & 1.785307 & -1.302246 \\
\hline $\mathrm{C}$ & -2.959535 & 2.871626 & -0.618193 \\
\hline $\mathrm{C}$ & -2.998467 & 2.862672 & 0.801242 \\
\hline $\mathrm{C}$ & -1.909875 & 3.398518 & 1.566123 \\
\hline $\mathrm{C}$ & -1.779425 & 2.625275 & 2.748682 \\
\hline $\mathrm{C}$ & -2.783318 & 1.578842 & 2.728798 \\
\hline $\mathrm{C}$ & -3.533233 & 1.744062 & 1.509704 \\
\hline $\mathrm{Sc}$ & -0.846776 & -0.091419 & 1.950089 \\
\hline $\mathrm{Sc}$ & -0.786503 & 0.181473 & -1.960497 \\
\hline $\mathrm{C}$ & 0.171846 & 0.674415 & 0.044670 \\
\hline $\mathrm{C}$ & 0.185604 & -0.589079 & -0.029448 \\
\hline
\end{tabular}

Product from bond 7 of $\mathrm{C}_{82}$

$\begin{array}{lrrr}\mathrm{C} & -1.61255100 & 2.40696400 & -3.28697700 \\ \mathrm{C} & -1.03638400 & 3.54938800 & -2.64246400 \\ \mathrm{C} & 0.39106200 & 3.74425600 & -2.62308900\end{array}$




\begin{tabular}{|c|c|c|c|}
\hline $\mathrm{C}$ & 0.74121600 & 4.38051500 & -1.36784200 \\
\hline $\mathrm{C}$ & 1.98547400 & 4.15750100 & -0.74805600 \\
\hline $\mathrm{C}$ & 1.97693400 & 4.25432100 & 0.66766000 \\
\hline $\mathrm{C}$ & 2.87645400 & 3.45438200 & 1.45720300 \\
\hline $\mathrm{C}$ & 2.18805800 & 2.96884700 & 2.62473500 \\
\hline $\mathrm{C}$ & 2.39884700 & 1.64256200 & 3.08246300 \\
\hline $\mathrm{C}$ & 1.28969700 & 1.04585000 & 3.71182700 \\
\hline $\mathrm{C}$ & 1.06982500 & -0.38705200 & 3.66997500 \\
\hline $\mathrm{C}$ & -0.35067000 & -0.62354700 & 3.71448200 \\
\hline $\mathrm{C}$ & -0.93302200 & -1.72736100 & 3.01011300 \\
\hline $\mathrm{C}$ & -2.20173300 & -1.56016700 & 2.42071300 \\
\hline $\mathrm{C}$ & -2.55304100 & -2.22053400 & 1.17611300 \\
\hline $\mathrm{C}$ & -3.39163400 & -1.35273600 & 0.45567600 \\
\hline $\mathrm{C}$ & -3.34451000 & -1.27987700 & -0.97839300 \\
\hline $\mathrm{C}$ & -3.61181700 & -0.00803700 & -1.59136800 \\
\hline $\mathrm{C}$ & -2.97999900 & 0.38079300 & -2.78445500 \\
\hline $\mathrm{C}$ & -2.75581400 & 1.81378100 & -2.71701700 \\
\hline $\mathrm{C}$ & -0.72593600 & 1.57709300 & -4.02806800 \\
\hline $\mathrm{C}$ & -0.94047500 & 0.12374500 & -4.09594500 \\
\hline $\mathrm{C}$ & -2.03119000 & -0.47570300 & -3.42421500 \\
\hline $\mathrm{C}$ & -1.80830300 & -1.75769800 & -2.86310300 \\
\hline $\mathrm{C}$ & -2.47677000 & -2.15683100 & -1.64763200 \\
\hline $\mathrm{C}$ & -1.59798000 & -3.03692200 & -0.91481100 \\
\hline $\mathrm{C}$ & -1.60732700 & -3.05402700 & 0.50195800 \\
\hline $\mathrm{C}$ & -0.37453900 & -3.31647100 & 1.14377000 \\
\hline $\mathrm{C}$ & -0.03111700 & -2.64945000 & 2.40905400 \\
\hline $\mathrm{C}$ & 1.35450700 & -2.42918400 & 2.44025400 \\
\hline $\mathrm{C}$ & 1.93849600 & -1.22996800 & 2.98469200 \\
\hline $\mathrm{C}$ & 3.13982600 & -0.65532500 & 2.35399600 \\
\hline $\mathrm{C}$ & 3.37277100 & 0.78617000 & 2.40735800 \\
\hline $\mathrm{C}$ & 4.14565900 & 1.37454400 & 1.37954100 \\
\hline $\mathrm{C}$ & 3.82687100 & 2.69164100 & 0.85383200 \\
\hline $\mathrm{C}$ & 3.93348600 & 2.67200100 & -0.59563900 \\
\hline $\mathrm{C}$ & 2.96115000 & 3.29451400 & -1.41249900 \\
\hline $\mathrm{C}$ & 2.59907200 & 2.64183900 & -2.66914500 \\
\hline $\mathrm{C}$ & 1.26596300 & 2.86688900 & -3.25499100 \\
\hline $\mathrm{C}$ & 0.65419300 & 1.83098800 & -4.04773500 \\
\hline $\mathrm{C}$ & 1.30367500 & 0.55258100 & -4.20187100 \\
\hline $\mathrm{C}$ & 0.32013100 & -0.50458400 & -4.14834100 \\
\hline $\mathrm{C}$ & 0.59871600 & -1.71326100 & -3.44112600 \\
\hline $\mathrm{C}$ & -0.51224200 & -2.34562800 & -2.85792100 \\
\hline $\mathrm{C}$ & -0.38166000 & -3.14120800 & -1.64679100 \\
\hline $\mathrm{C}$ & 0.86052900 & -3.30746600 & -1.01201800 \\
\hline $\mathrm{C}$ & 0.81163100 & -3.49223500 & 0.40291500 \\
\hline
\end{tabular}




\begin{tabular}{|c|c|c|c|}
\hline $\mathrm{C}$ & 1.89108900 & -3.01951700 & 1.23836600 \\
\hline $\mathrm{C}$ & 3.01203200 & -2.50764100 & 0.67641800 \\
\hline $\mathrm{C}$ & 3.70938500 & -1.37759100 & 1.28839000 \\
\hline $\mathrm{C}$ & 4.42876100 & -0.74284800 & 0.23776000 \\
\hline $\mathrm{C}$ & 4.64549400 & 0.61862900 & 0.28159100 \\
\hline $\mathrm{C}$ & 4.51514400 & 1.41693100 & -0.93381800 \\
\hline $\mathrm{C}$ & 4.17500100 & 0.80720000 & -2.12340000 \\
\hline $\mathrm{C}$ & 3.24725700 & 1.43662800 & -2.99942400 \\
\hline $\mathrm{C}$ & 2.54078700 & 0.35700200 & -3.68675000 \\
\hline $\mathrm{C}$ & 2.89609300 & -0.91574000 & -3.08278500 \\
\hline $\mathrm{C}$ & 1.92042600 & -1.90864900 & -2.83784300 \\
\hline $\mathrm{C}$ & 2.05153800 & -2.70768000 & -1.62134700 \\
\hline $\mathrm{C}$ & 3.14538300 & -2.43546700 & -0.76865700 \\
\hline $\mathrm{C}$ & 4.07758000 & -1.38962400 & -1.02413800 \\
\hline $\mathrm{C}$ & 3.95404300 & -0.63627300 & -2.17119100 \\
\hline $\mathrm{C}$ & -1.55159300 & 4.02656300 & -1.41963900 \\
\hline $\mathrm{C}$ & -0.44434600 & 4.56052200 & -0.61984600 \\
\hline $\mathrm{C}$ & -0.45291000 & 4.51355800 & 0.78228300 \\
\hline $\mathrm{C}$ & 0.77561300 & 4.39189200 & 1.41309500 \\
\hline $\mathrm{C}$ & 0.90820700 & 3.58404500 & 2.64249700 \\
\hline $\mathrm{C}$ & -0.19447500 & 2.93891600 & 3.18170200 \\
\hline $\mathrm{C}$ & 0.02878100 & 1.68221600 & 3.76484400 \\
\hline $\mathrm{C}$ & -0.99383500 & 0.63018000 & 3.75355300 \\
\hline $\mathrm{C}$ & -2.23099600 & 0.83760000 & 3.09256300 \\
\hline $\mathrm{C}$ & -2.84659400 & -0.28841200 & 2.44829200 \\
\hline $\mathrm{C}$ & -3.59288800 & -0.15348300 & 1.25001100 \\
\hline $\mathrm{C}$ & -3.71499400 & 1.10133000 & 0.62526800 \\
\hline $\mathrm{C}$ & -3.81138400 & 1.17730900 & -0.77627300 \\
\hline $\mathrm{C}$ & -3.27015500 & 2.28726600 & -1.47378500 \\
\hline $\mathrm{C}$ & -2.64930600 & 3.38675800 & -0.79040100 \\
\hline $\mathrm{C}$ & -2.64624800 & 3.36359900 & 0.62097100 \\
\hline $\mathrm{C}$ & -1.76342900 & 4.22724600 & 1.50795400 \\
\hline $\mathrm{C}$ & -1.62072600 & 3.35641700 & 2.83734000 \\
\hline $\mathrm{C}$ & -2.44193900 & 2.11394000 & 2.52664200 \\
\hline $\mathrm{C}$ & -3.14412900 & 2.24527900 & 1.31451500 \\
\hline $\mathrm{C}$ & -2.46158200 & 5.59685100 & 1.80215500 \\
\hline $\mathrm{C}$ & -1.82130900 & 6.25192200 & 2.99605300 \\
\hline $\mathrm{C}$ & -1.70253700 & 5.52564700 & 4.10667800 \\
\hline $\mathrm{C}$ & -2.21673800 & 4.11139000 & 4.07232300 \\
\hline $\mathrm{H}$ & -2.41555000 & 6.21813900 & 0.90425400 \\
\hline $\mathrm{H}$ & -3.52335500 & 5.40176700 & 2.00740700 \\
\hline $\mathrm{H}$ & -1.46743400 & 7.27587900 & 2.92937100 \\
\hline $\mathrm{H}$ & -1.24617100 & 5.91537100 & 5.01114600 \\
\hline $\mathrm{H}$ & -3.31157500 & 4.11329100 & 3.97652700 \\
\hline
\end{tabular}




$$
\begin{array}{llll}
\mathrm{H} & -1.97308900 & 3.55218800 & 4.97909900
\end{array}
$$

TS from bond 7 of $\mathrm{C}_{82}$

\begin{tabular}{|c|c|c|c|}
\hline $\mathrm{C}$ & -1.56395100 & 2.31005900 & -3.29397700 \\
\hline $\mathrm{C}$ & -0.98523300 & 3.45208900 & -2.64656900 \\
\hline $\mathrm{C}$ & 0.44239100 & 3.65457100 & -2.63091500 \\
\hline $\mathrm{C}$ & 0.79174000 & 4.30085600 & -1.37673300 \\
\hline $\mathrm{C}$ & 2.03942800 & 4.07558400 & -0.75163300 \\
\hline $\mathrm{C}$ & 2.03913000 & 4.17773200 & 0.66302100 \\
\hline $\mathrm{C}$ & 2.93764000 & 3.37429400 & 1.45292800 \\
\hline $\mathrm{C}$ & 2.24923100 & 2.88950000 & 2.62277000 \\
\hline $\mathrm{C}$ & 2.45051900 & 1.56058900 & 3.07600500 \\
\hline $\mathrm{C}$ & 1.33822900 & 0.95984100 & 3.70845600 \\
\hline $\mathrm{C}$ & 1.11702300 & -0.47601600 & 3.65594400 \\
\hline $\mathrm{C}$ & -0.30489000 & -0.71126700 & 3.69066000 \\
\hline $\mathrm{C}$ & -0.88964500 & -1.81638000 & 2.98673100 \\
\hline $\mathrm{C}$ & -2.15620700 & -1.65227800 & 2.40435300 \\
\hline $\mathrm{C}$ & -2.50056500 & -2.30961000 & 1.15083700 \\
\hline $\mathrm{C}$ & -3.34051100 & -1.44898000 & 0.43353800 \\
\hline $\mathrm{C}$ & -3.29248400 & -1.38013100 & -1.00104400 \\
\hline $\mathrm{C}$ & -3.55904300 & -0.10591200 & -1.60998400 \\
\hline $\mathrm{C}$ & -2.92377000 & 0.28331800 & -2.79478900 \\
\hline $\mathrm{C}$ & -2.70707600 & 1.72251600 & -2.73123400 \\
\hline $\mathrm{C}$ & -0.67304800 & 1.48115500 & -4.03761900 \\
\hline $\mathrm{C}$ & -0.88569900 & 0.02739600 & -4.11101300 \\
\hline $\mathrm{C}$ & -1.97480300 & -0.57375400 & -3.44125700 \\
\hline $\mathrm{C}$ & -1.75308800 & -1.85410500 & -2.88086000 \\
\hline $\mathrm{C}$ & -2.42736800 & -2.25700000 & -1.66985400 \\
\hline $\mathrm{C}$ & -1.54422500 & -3.13198500 & -0.93587500 \\
\hline $\mathrm{C}$ & -1.55377800 & -3.14966300 & 0.47888400 \\
\hline $\mathrm{C}$ & -0.32346700 & -3.41119800 & 1.12262400 \\
\hline $\mathrm{C}$ & 0.01672200 & -2.74033400 & 2.38718400 \\
\hline $\mathrm{C}$ & 1.39840200 & -2.51858100 & 2.42125700 \\
\hline $\mathrm{C}$ & 1.98250700 & -1.31543700 & 2.96799900 \\
\hline $\mathrm{C}$ & 3.18611600 & -0.74112000 & 2.34091500 \\
\hline $\mathrm{C}$ & 3.41951900 & 0.70092600 & 2.39680400 \\
\hline $\mathrm{C}$ & 4.19631200 & 1.28735700 & 1.37087100 \\
\hline $\mathrm{C}$ & 3.88166000 & 2.60522900 & 0.84644200 \\
\hline $\mathrm{C}$ & 3.98543100 & 2.58460800 & -0.60247400 \\
\hline $\mathrm{C}$ & 3.01072300 & 3.20693900 & -1.41658200 \\
\hline $\mathrm{C}$ & 2.64906300 & 2.55275300 & -2.67326900 \\
\hline $\mathrm{C}$ & 1.31471400 & 2.77568500 & -3.25799200 \\
\hline $\mathrm{C}$ & 0.70307800 & 1.73706900 & -4.05497400 \\
\hline $\mathrm{C}$ & 1.35653700 & 0.45967800 & -4.21277800 \\
\hline
\end{tabular}




\begin{tabular}{|c|c|c|c|}
\hline $\mathrm{C}$ & 0.37612600 & -0.59962400 & -4.16520000 \\
\hline $\mathrm{C}$ & 0.65488500 & -1.80769800 & -3.45822000 \\
\hline $\mathrm{C}$ & -0.45497800 & -2.44308400 & -2.87663200 \\
\hline $\mathrm{C}$ & -0.32540000 & -3.23777400 & -1.66774400 \\
\hline $\mathrm{C}$ & 0.91517000 & -3.40267800 & -1.02973400 \\
\hline $\mathrm{C}$ & 0.86478200 & -3.58845400 & 0.38437100 \\
\hline $\mathrm{C}$ & 1.94014900 & -3.11110900 & 1.22116900 \\
\hline $\mathrm{C}$ & 3.06071900 & -2.59656100 & 0.66259900 \\
\hline $\mathrm{C}$ & 3.75590300 & -1.46359100 & 1.27656400 \\
\hline $\mathrm{C}$ & 4.47873700 & -0.83106000 & 0.22792400 \\
\hline $\mathrm{C}$ & 4.69518500 & 0.53029800 & 0.27282400 \\
\hline $\mathrm{C}$ & 4.56565000 & 1.32874100 & -0.94190100 \\
\hline $\mathrm{C}$ & 4.22648500 & 0.71920000 & -2.13161600 \\
\hline $\mathrm{C}$ & 3.29688700 & 1.34859600 & -3.00463900 \\
\hline $\mathrm{C}$ & 2.59242200 & 0.26707100 & -3.69542300 \\
\hline $\mathrm{C}$ & 2.95115200 & -1.00538900 & -3.09417900 \\
\hline $\mathrm{C}$ & 1.97682500 & -2.00081500 & -2.85200600 \\
\hline $\mathrm{C}$ & 2.10705700 & -2.79965100 & -1.63673300 \\
\hline $\mathrm{C}$ & 3.19893800 & -2.52534500 & -0.78154200 \\
\hline $\mathrm{C}$ & 4.13079800 & -1.47891000 & -1.03526300 \\
\hline $\mathrm{C}$ & 4.00796700 & -0.72540000 & -2.18158200 \\
\hline $\mathrm{C}$ & -1.49642500 & 3.92515100 & -1.42411800 \\
\hline $\mathrm{C}$ & -0.39347700 & 4.48035800 & -0.63696100 \\
\hline $\mathrm{C}$ & -0.40097300 & 4.42534300 & 0.77314100 \\
\hline $\mathrm{C}$ & 0.83611700 & 4.31259800 & 1.41471200 \\
\hline $\mathrm{C}$ & 0.96734700 & 3.50998900 & 2.63548500 \\
\hline $\mathrm{C}$ & -0.14384800 & 2.85042400 & 3.16981400 \\
\hline $\mathrm{C}$ & 0.07917800 & 1.58932000 & 3.76339500 \\
\hline $\mathrm{C}$ & -0.94338900 & 0.54178100 & 3.72477100 \\
\hline $\mathrm{C}$ & -2.18956000 & 0.74829200 & 3.06723000 \\
\hline $\mathrm{C}$ & -2.81088800 & -0.38318700 & 2.43434200 \\
\hline $\mathrm{C}$ & -3.54761300 & -0.24676700 & 1.23211400 \\
\hline $\mathrm{C}$ & -3.69886400 & 1.01843700 & 0.61859400 \\
\hline $\mathrm{C}$ & -3.76480000 & 1.08393300 & -0.79228800 \\
\hline $\mathrm{C}$ & -3.23277700 & 2.19596300 & -1.49015100 \\
\hline $\mathrm{C}$ & -2.60435500 & 3.28726900 & -0.79711900 \\
\hline $\mathrm{C}$ & -2.62815500 & 3.26749600 & 0.60977300 \\
\hline $\mathrm{C}$ & -1.59321700 & 3.91962700 & 1.44377200 \\
\hline $\mathrm{C}$ & -1.46494400 & 3.14051200 & 2.62764600 \\
\hline $\mathrm{C}$ & -2.42408500 & 2.02021900 & 2.51134500 \\
\hline $\mathrm{C}$ & -3.17700600 & 2.16720300 & 1.31696300 \\
\hline $\mathrm{C}$ & -2.70942000 & 5.90478100 & 1.98879100 \\
\hline $\mathrm{C}$ & -1.91854700 & 6.31628700 & 3.02830300 \\
\hline $\mathrm{C}$ & -1.77477000 & 5.55778600 & 4.21841700 \\
\hline
\end{tabular}




$\begin{array}{llll}\mathrm{C} & -2.42041200 & 4.36618600 & 4.41191500 \\ \mathrm{H} & -2.70488200 & 6.43850600 & 1.04281600 \\ \mathrm{H} & -3.54844700 & 5.23378100 & 2.14329900 \\ \mathrm{H} & -1.23366100 & 7.14501400 & 2.86858800 \\ \mathrm{H} & -0.98615500 & 5.84388100 & 4.90958100 \\ \mathrm{H} & -3.34176300 & 4.12437400 & 3.89184500 \\ \mathrm{H} & -2.19200100 & 3.74559200 & 5.27342600\end{array}$

Product from bond 19 of $\mathrm{Sc}_{2} \mathrm{C}_{2} @ \mathrm{C}_{82}$

$\begin{array}{lrrr}\text { C } & -1.93525600 & 1.90516000 & -3.19647700 \\ \mathrm{C} & -1.32624100 & 2.98412600 & -2.48799300 \\ \mathrm{C} & 0.11037600 & 3.17961800 & -2.45470600 \\ \mathrm{C} & 0.44760000 & 3.81903300 & -1.23317900 \\ \mathrm{C} & 1.68633500 & 3.54860600 & -0.58817800 \\ \mathrm{C} & 1.69226100 & 3.65553600 & 0.82193800 \\ \mathrm{C} & 2.57553800 & 2.84840000 & 1.60819300 \\ \mathrm{C} & 1.89099500 & 2.37167900 & 2.75596800 \\ \mathrm{C} & 2.07126700 & 1.03881000 & 3.16954300 \\ \mathrm{C} & 0.92883000 & 0.43530000 & 3.79321400 \\ \mathrm{C} & 0.70694800 & -0.96898400 & 3.77075100 \\ \mathrm{C} & -0.70122900 & -1.21467200 & 3.94316500 \\ \mathrm{C} & -1.27883700 & -2.31958400 & 3.20345300 \\ \mathrm{C} & -2.54804600 & -2.09610400 & 2.59247800 \\ \mathrm{C} & -2.89438900 & -2.71776500 & 1.35488000 \\ \mathrm{C} & -3.75683700 & -1.82957900 & 0.63795600 \\ \mathrm{C} & -3.70832800 & -1.76407300 & -0.78153700 \\ \mathrm{C} & -3.95258300 & -0.49973000 & -1.39115200 \\ \mathrm{C} & -3.29603000 & -0.12438700 & -2.60709600 \\ \mathrm{C} & -3.06812100 & 1.30655000 & -2.55936700 \\ \mathrm{C} & -1.08477600 & 1.04996100 & -3.99578800 \\ \mathrm{C} & -1.31765400 & -0.41016300 & -4.04452200 \\ \mathrm{C} & -2.40032400 & -1.00528500 & -3.29275000 \\ \mathrm{C} & -2.16235200 & -2.26221000 & -2.65919800 \\ \mathrm{C} & -2.83297300 & -2.65679100 & -1.45950600 \\ \mathrm{C} & -1.96853300 & -3.54441300 & -0.74180900 \\ \mathrm{C} & -1.97240100 & -3.56903000 & 0.67795600 \\ \mathrm{C} & -0.74587100 & -3.85461300 & 1.32398300 \\ \mathrm{C} & -0.40294900 & -3.22727200 & 2.56792500 \\ \mathrm{C} & 1.03419500 & -3.03095400 & 2.58276400 \\ \mathrm{C} & 1.61436300 & -1.85117100 & 3.08601400 \\ \mathrm{C} & 2.81252600 & -1.29437700 & 2.47456400 \\ & 3.04817400 & 0.17965100 & 2.51851000 \\ \mathrm{C} & 3.82623500 & 0.74818100 & 1.51495600 \\ \mathrm{C} & & & 0.99930000\end{array}$




\begin{tabular}{|c|c|c|c|}
\hline $\mathrm{C}$ & 3.60536800 & 2.01053400 & -0.43643500 \\
\hline $\mathrm{C}$ & 2.64186800 & 2.66272400 & -1.24944400 \\
\hline $\mathrm{C}$ & 2.29602700 & 2.02670300 & -2.50854900 \\
\hline $\mathrm{C}$ & 0.97965100 & 2.26623700 & -3.08469600 \\
\hline $\mathrm{C}$ & 0.34873800 & 1.24956200 & -3.88959000 \\
\hline $\mathrm{C}$ & 0.97479700 & -0.02970400 & -3.99333600 \\
\hline $\mathrm{C}$ & -0.01934100 & -1.05438400 & -3.96793500 \\
\hline $\mathrm{C}$ & 0.25597400 & -2.26573400 & -3.23537900 \\
\hline $\mathrm{C}$ & -0.86162600 & -2.90112000 & -2.65675400 \\
\hline $\mathrm{C}$ & -0.75171600 & -3.68899900 & -1.48022100 \\
\hline $\mathrm{C}$ & 0.50228900 & -3.85916700 & -0.83166200 \\
\hline $\mathrm{C}$ & 0.45998100 & -4.05210200 & 0.56962700 \\
\hline $\mathrm{C}$ & 1.54350300 & -3.61896900 & 1.39759000 \\
\hline $\mathrm{C}$ & 2.68486400 & -3.09758700 & 0.82982200 \\
\hline $\mathrm{C}$ & 3.38354100 & -2.01014700 & 1.42585700 \\
\hline $\mathrm{C}$ & 4.41844200 & -1.51914700 & 0.42351200 \\
\hline $\mathrm{C}$ & 4.66751000 & 0.02548800 & 0.47350600 \\
\hline $\mathrm{C}$ & 4.20847300 & 0.77250300 & -0.77896400 \\
\hline $\mathrm{C}$ & 3.89425700 & 0.18315100 & -1.96559300 \\
\hline $\mathrm{C}$ & 2.94769200 & 0.80716500 & -2.83579600 \\
\hline $\mathrm{C}$ & 2.25237800 & -0.25016900 & -3.50900200 \\
\hline $\mathrm{C}$ & 2.57694600 & -1.51117700 & -2.91193000 \\
\hline $\mathrm{C}$ & 1.57553500 & -2.48731100 & -2.65843300 \\
\hline $\mathrm{C}$ & 1.69402900 & -3.27978500 & -1.44713500 \\
\hline $\mathrm{C}$ & 2.80280100 & -3.01275200 & -0.60229900 \\
\hline $\mathrm{C}$ & 3.76372800 & -2.00262100 & -0.86961900 \\
\hline $\mathrm{C}$ & 3.66077000 & -1.26960500 & -2.01181700 \\
\hline $\mathrm{C}$ & -1.85152000 & 3.49305100 & -1.25927900 \\
\hline $\mathrm{C}$ & -0.76022300 & 4.01449000 & -0.49308800 \\
\hline $\mathrm{C}$ & -0.77144800 & 3.95129500 & 0.92592300 \\
\hline $\mathrm{C}$ & 0.47721000 & 3.79915700 & 1.57491000 \\
\hline $\mathrm{C}$ & 0.59498200 & 3.01669100 & 2.77136500 \\
\hline $\mathrm{C}$ & -0.52831300 & 2.37988400 & 3.33038800 \\
\hline $\mathrm{C}$ & -0.33402500 & 1.09051100 & 3.95522400 \\
\hline $\mathrm{C}$ & -1.37727600 & 0.07979500 & 4.07291300 \\
\hline $\mathrm{C}$ & -2.63694300 & 0.31222800 & 3.36489400 \\
\hline $\mathrm{C}$ & -3.19797500 & -0.80086700 & 2.65250100 \\
\hline $\mathrm{C}$ & -3.93136600 & -0.64658200 & 1.42304200 \\
\hline $\mathrm{C}$ & -4.09271200 & 0.62777100 & 0.81400300 \\
\hline $\mathrm{C}$ & -4.14507400 & 0.68103500 & -0.60770900 \\
\hline $\mathrm{C}$ & -3.58627300 & 1.78993200 & -1.31502000 \\
\hline $\mathrm{C}$ & -2.96690900 & 2.87547500 & -0.62736100 \\
\hline $\mathrm{C}$ & -3.01105400 & 2.86549300 & 0.79184600 \\
\hline $\mathrm{C}$ & -1.92449800 & 3.39987400 & 1.56058000 \\
\hline
\end{tabular}




$\begin{array}{lccc}\mathrm{C} & -1.79761000 & 2.62279700 & 2.74290700 \\ \mathrm{C} & -2.80221500 & 1.57801700 & 2.71806500 \\ \mathrm{C} & -3.54891100 & 1.74612500 & 1.49748000 \\ \mathrm{Sc} & -0.85985900 & -0.10763100 & 1.94227200 \\ \mathrm{Sc} & -0.79120500 & 0.18710800 & -1.96375800 \\ \mathrm{C} & 0.15665400 & 0.67843000 & 0.05030800 \\ \mathrm{C} & 0.19024900 & -0.58348000 & -0.04105700 \\ \mathrm{C} & 6.18021000 & 0.34551900 & 0.72297300 \\ \mathrm{C} & 7.04589900 & -0.45628900 & -0.20530100 \\ \mathrm{C} & 6.83443500 & -1.77139400 & -0.24605200 \\ \mathrm{C} & 5.75073200 & -2.31476900 & 0.63937900 \\ \mathrm{H} & 6.33023700 & 1.42245100 & 0.61280900 \\ \mathrm{H} & 6.40798100 & 0.09478000 & 1.76815900 \\ \mathrm{H} & 7.79254900 & 0.03624700 & -0.82028100 \\ \mathrm{H} & 7.39485100 & -2.43503100 & -0.89706000 \\ \mathrm{H} & 6.03490000 & -2.21227300 & 1.69591500 \\ \mathrm{H} & 5.55472600 & -3.37518500 & 0.46224300\end{array}$

TS from bond 19 of $\mathrm{Sc}_{2} \mathrm{C}_{2} @ \mathrm{C}_{82}$

$\begin{array}{lrrr}\mathrm{C} & -2.15775400 & 2.04695200 & -3.24327600 \\ \mathrm{C} & -1.54250600 & 3.12100500 & -2.53362400 \\ \mathrm{C} & -0.10407000 & 3.30510500 & -2.49693300 \\ \mathrm{C} & 0.23427900 & 3.94019600 & -1.27327200 \\ \mathrm{C} & 1.46985300 & 3.66250200 & -0.62522500 \\ \mathrm{C} & 1.47087700 & 3.76676600 & 0.78526600 \\ \mathrm{C} & 2.34613700 & 2.95307800 & 1.57382000 \\ \mathrm{C} & 1.65218500 & 2.48015300 & 2.72364500 \\ \mathrm{C} & 1.81879400 & 1.14905500 & 3.14837200 \\ \mathrm{C} & 0.66868200 & 0.55407200 & 3.76608200 \\ \mathrm{C} & 0.43513700 & -0.84949500 & 3.74086600 \\ \mathrm{C} & -0.97645700 & -1.08302700 & 3.90109100 \\ \mathrm{C} & -1.55723300 & -2.18205700 & 3.15659700 \\ \mathrm{C} & -2.82339200 & -1.94973000 & 2.54118900 \\ \mathrm{C} & -3.16938200 & -2.56756100 & 1.30304200 \\ \mathrm{C} & -4.02272400 & -1.67279400 & 0.58293300 \\ \mathrm{C} & -3.96869200 & -1.60718600 & -0.83635000 \\ \mathrm{C} & -4.20201200 & -0.34107000 & -1.44636400 \\ \mathrm{C} & -3.53767500 & 0.02934200 & -2.65946300 \\ \mathrm{C} & -3.29817600 & 1.45764700 & -2.61025600 \\ \mathrm{C} & -1.31220500 & 1.18566900 & -4.04043200 \\ \mathrm{C} & -1.55714900 & -0.27220100 & -4.09170000 \\ \mathrm{C} & -2.64674300 & -0.85852000 & -3.34335700 \\ \mathrm{C} & -2.42016000 & -2.11677200 & -2.70904700 \\ \mathrm{C} & -3.09765600 & -2.50624700 & -1.51191500 \\ & & & \\ & & 513 & \\ & & & \\ & & & \end{array}$




\begin{tabular}{|c|c|c|c|}
\hline $\mathrm{C}$ & -2.24137400 & -3.39976200 & -0.79161000 \\
\hline $\mathrm{C}$ & -2.25015100 & -3.42502500 & 0.62866700 \\
\hline $\mathrm{C}$ & -1.02942700 & -3.72015900 & 1.27797700 \\
\hline $\mathrm{C}$ & -0.68503600 & -3.09548400 & 2.52363600 \\
\hline $\mathrm{C}$ & 0.75273200 & -2.91183700 & 2.54610000 \\
\hline $\mathrm{C}$ & 1.33800800 & -1.73999500 & 3.06137300 \\
\hline $\mathrm{C}$ & 2.53850000 & -1.18275500 & 2.45240300 \\
\hline $\mathrm{C}$ & 2.78347900 & 0.27433400 & 2.49778200 \\
\hline $\mathrm{C}$ & 3.57257900 & 0.84104500 & 1.48398500 \\
\hline $\mathrm{C}$ & 3.27587200 & 2.14506800 & 0.96518400 \\
\hline $\mathrm{C}$ & 3.38490800 & 2.12532900 & -0.46812400 \\
\hline $\mathrm{C}$ & 2.42525000 & 2.77367100 & -1.28525300 \\
\hline $\mathrm{C}$ & 2.07569000 & 2.13537100 & -2.54781600 \\
\hline $\mathrm{C}$ & 0.75934000 & 2.38430600 & -3.12367700 \\
\hline $\mathrm{C}$ & 0.12239900 & 1.37393200 & -3.93124500 \\
\hline $\mathrm{C}$ & 0.73896800 & 0.08973400 & -4.03749100 \\
\hline $\mathrm{C}$ & -0.26412700 & -0.92700600 & -4.01259000 \\
\hline $\mathrm{C}$ & 0.00008400 & -2.13932100 & -3.27808000 \\
\hline $\mathrm{C}$ & -1.12424800 & -2.76657600 & -2.70372200 \\
\hline $\mathrm{C}$ & -1.02436500 & -3.55452500 & -1.52636000 \\
\hline $\mathrm{C}$ & 0.22627200 & -3.73798700 & -0.87484900 \\
\hline $\mathrm{C}$ & 0.17749600 & -3.92846700 & 0.52660800 \\
\hline $\mathrm{C}$ & 1.26247700 & -3.50527200 & 1.35788100 \\
\hline $\mathrm{C}$ & 2.41104200 & -3.00552800 & 0.79066300 \\
\hline $\mathrm{C}$ & 3.10891500 & -1.90700900 & 1.39253300 \\
\hline $\mathrm{C}$ & 3.96266300 & -1.31933000 & 0.36329900 \\
\hline $\mathrm{C}$ & 4.19959900 & 0.07592600 & 0.40920800 \\
\hline $\mathrm{C}$ & 3.96292000 & 0.86709700 & -0.81199800 \\
\hline $\mathrm{C}$ & 3.65304200 & 0.27520200 & -2.00734600 \\
\hline $\mathrm{C}$ & 2.71865500 & 0.91273800 & -2.88236600 \\
\hline $\mathrm{C}$ & 2.01518100 & -0.14099800 & -3.55814500 \\
\hline $\mathrm{C}$ & 2.32890200 & -1.40727300 & -2.96035000 \\
\hline $\mathrm{C}$ & 1.31903000 & -2.37391700 & -2.70136700 \\
\hline $\mathrm{C}$ & 1.42744000 & -3.17334400 & -1.48808200 \\
\hline $\mathrm{C}$ & 2.53565300 & -2.92857900 & -0.63911800 \\
\hline $\mathrm{C}$ & 3.49552100 & -1.90640800 & -0.90445100 \\
\hline $\mathrm{C}$ & 3.40950600 & -1.16844500 & -2.05447900 \\
\hline $\mathrm{C}$ & -2.06763200 & 3.63339100 & -1.30678500 \\
\hline $\mathrm{C}$ & -0.97361500 & 4.14410700 & -0.53678000 \\
\hline $\mathrm{C}$ & -0.99011600 & 4.08068600 & 0.88262700 \\
\hline $\mathrm{C}$ & 0.25372800 & 3.91820700 & 1.53489000 \\
\hline $\mathrm{C}$ & 0.36145000 & 3.13374100 & 2.73196300 \\
\hline $\mathrm{C}$ & -0.76981400 & 2.50773800 & 3.28880700 \\
\hline $\mathrm{C}$ & -0.59042100 & 1.21924300 & 3.91873300 \\
\hline
\end{tabular}




$\begin{array}{lrrc}\text { C } & -1.64305500 & 0.21650400 & 4.02924900 \\ \mathrm{C} & -2.89753100 & 0.45897000 & 3.31539900 \\ \mathrm{C} & -3.46334100 & -0.64917200 & 2.59934600 \\ \mathrm{C} & -4.19123300 & -0.48891100 & 1.36741300 \\ \mathrm{C} & -4.34108200 & 0.78684300 & 0.75789700 \\ \mathrm{C} & -4.38899200 & 0.84112500 & -0.66332000 \\ \mathrm{C} & -3.81726800 & 1.94527000 & -1.36834800 \\ \mathrm{C} & -3.19019100 & 3.02485400 & -0.67860500 \\ \mathrm{C} & -3.23927600 & 3.01469500 & 0.74056200 \\ \mathrm{C} & -2.15092300 & 3.53931300 & 1.51357000 \\ \mathrm{C} & -2.03549100 & 2.76224200 & 2.69596200 \\ \mathrm{C} & -3.04904600 & 1.72591500 & 2.66731400 \\ \mathrm{C} & -3.78962300 & 1.90033100 & 1.44386500 \\ \mathrm{Sc} & -1.11842700 & 0.03457400 & 1.90033800 \\ \mathrm{Sc} & -1.02907800 & 0.31469200 & -2.00910700 \\ \mathrm{C} & -0.08476500 & 0.79818700 & 0.00489300 \\ \mathrm{C} & -0.07407900 & -0.46510000 & -0.07406600 \\ \mathrm{C} & 6.38665300 & 0.41792600 & 0.79215100 \\ \mathrm{C} & 6.92974900 & -0.38114600 & -0.18683400 \\ \mathrm{C} & 6.69432800 & -1.77196200 & -0.22080300 \\ \mathrm{C} & 5.90797600 & -2.39164700 & 0.72193300 \\ \mathrm{H} & 6.46623000 & 1.49937000 & 0.72824700 \\ \mathrm{H} & 6.18091800 & 0.02940800 & 1.78370300 \\ \mathrm{H} & 7.36051300 & 0.08745900 & -1.06770300 \\ \mathrm{H} & 6.95476800 & -2.31501700 & -1.12535300 \\ \mathrm{H} & 5.82808700 & -2.00102900 & 1.73056400 \\ \mathrm{H} & 5.62499500 & -3.43410500 & 0.60703100\end{array}$

